\title{
Specific Dark Matter signatures from hidden U(1)
}

\author{
Yann Mambrini* \\ Laboratoire de Physique Théorique, Université Paris-Sud, F-91405 Orsay, France \\ E-mail: yann.mambrini@th.u-psud.fr
}

Several constructions motivate the existence of a dark $U(1)_{D}$ gauge boson which interacts with the Standard Model only through its kinetic mixing or loop induced processes. We describe two typical examples with specific signatures in particular we show that a region with relatively light WIMPS, $M_{Z_{D}} \lesssim 40 \mathrm{GeV}$ and a kinetic mixing $10^{-4} \lesssim \delta \lesssim 10^{-3}$ is not yet excluded by the last experimental data and seems to give promising signals in a near future. We also show that conditions from anomaly cancelation generate tri-vector couplings $Z^{\prime} Z \gamma$ leading to a specific gamma ray line observable by FERMI telescope.

The XIXth International Workshop on High Energy Physics and Quantum Field Theory, QFTHEP2010 September 08-15, 2010

Golitsyno, Moscow, Russia

\footnotetext{
* Speaker.
} 


\section{Introduction}

Neutral gauge sectors with an additional dark $U(1)_{D}$ symmetry in addition to the Standard Model (SM) hypercharge $U(1)_{Y}$ and an associated $Z_{D}$ are among the best motivated extensions of the SM, and give the possibility that a dark matter candidate lies within this new gauge sector of the theory [1]. The new vector boson $Z_{D}$ can interact with the SM, even if no SM fermions are directly charged under the additional gauge symmetry. This interaction can occurs via mixed kinetic terms between the SM's hypercharge field strength and the new abelian field strength [2, 3, 4] or through couplings generated by counter-term to preserve the anomaly cancelation condition [5, 6]. Whereas the former couplings can give significant signals in direct detection experiment even fitting the last DAMA [7] or COGENT [8] excesses [9, 10, 11], the latter can give rise to a gamma-ray line observable in satellite telescopes [12, 13, 14, 15]

\section{The dark kinetic mixing}

The matter content of any dark $U(1)_{D}$ extension of the SM can be decomposed into three families of particles:

- The Visible sector is made of particles which are charged under the SM gauge group $S U(3) \times$ $S U(2) \times U(1)_{Y}$ but not charged under $U(1)_{D}$ (hence the dark denomination for this gauge group)

- the Dark sector is composed by the particles charged under $U(1)_{D}$ but neutral with respect of the SM gauge symmetries. The dark matter $\left(\psi_{0}\right)$ candidate is the lightest particle of the dark sector

- The Hybrid sector contains states with SM and $U(1)_{D}$ quantum numbers. These states are fundamental because they act as a portal between the two previous sector through the kinetic mixing they induce at loop order.

From these considerations, it is easy to build the effective lagrangian generated at one loop :

$$
\begin{aligned}
\mathscr{L} & =\mathscr{L}_{\mathrm{SM}}-\frac{1}{4} \tilde{B}_{\mu v} \tilde{B}^{\mu v}-\frac{1}{4} \tilde{X}_{\mu v} \tilde{X}^{\mu v}-\frac{\delta}{2} \tilde{B}_{\mu v} \tilde{X}^{\mu v} \\
& +i \sum_{i} \psi_{i} \gamma^{\mu} D_{\mu} \psi_{i}+i \sum_{j} \Psi_{j} \gamma^{\mu} D_{\mu} \Psi_{j}
\end{aligned}
$$

$B_{\mu}$ being the gauge field for the hypercharge, $X_{\mu}$ the gauge field of $U(1)_{D}$ and $\psi_{i}$ the particles from the hidden sector, $\Psi_{j}$ the particles from the hybrid sector, $D_{\mu}=\partial_{\mu}-i\left(q_{Y} \tilde{g}_{Y} \tilde{B}_{\mu}+q_{D} \tilde{g}_{D} \tilde{X}_{\mu}+\right.$ $\left.g T^{a} W_{\mu}^{a}\right), T^{a}$ being the $S U(2)$ generators, and

$$
\delta=\frac{\tilde{g}_{Y} \tilde{g}_{D}}{16 \pi^{2}} \sum_{j} q_{Y}^{j} q_{D}^{j} \log \left(\frac{m_{j}^{2}}{M_{j}^{2}}\right)
$$

with $m_{j}$ and $M_{j}$ being hybrid mass states [3]. Notice that the sum is on all the hybrid states, as they are the only ones which can contribute to the $Y_{\mu} X_{\mu}$ propagator. After diagonalization of the current eigenstates, one makes the gauge kinetic terms of Eq.(2.1) diagonal and canonical. 

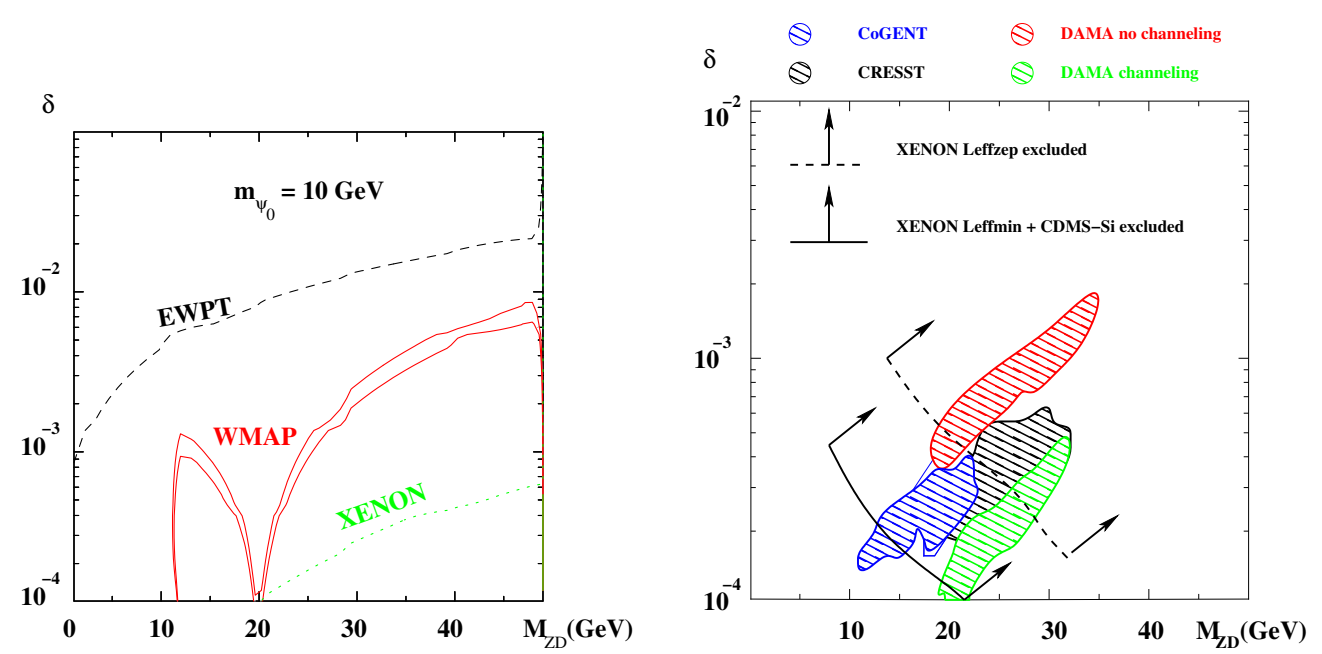

Figure 1: Left : example of allowed parameter space for $m_{\psi_{0}}=10 \mathrm{GeV}$ in the $\left(M_{Z_{D}}, \delta\right.$ ) plane (left). The points between the full-red region respect the $5 \sigma$ WMAP constraint, the points below the dashed-black line do not exceed accelerator data on precision tests, and the points above the dotted-green line are excluded by XENON100 data. Right: parameter space allowed within $90 \%$ of C.L. for the CoGeNT signal (blue), DAMA without channeling (red), with channeling (green), CRESST (black), and the exclusion region depending on the hypothesis concerning $L_{e f f}$.

We show in Fig.1 (left) the points that fulfill the WMAP $5 \sigma$ bound [16] on $\Omega_{\mathrm{DM}}$ for $m_{\psi_{0}}=$ $10 \mathrm{GeV}$ in the $\left(M_{Z_{D}}, \delta\right)$ plane. One can clearly see the $Z_{D}$-pole region when $M_{Z_{D}} \sim m_{\psi_{0}}$. One important point is that for a given $M_{Z_{D}}$ and $m_{\psi_{0}}$, there exists a unique solution $\delta$ (up to the very small uncertainties at $5 \sigma)$ fulfilling WMAP constraints : from 3 parameters $\left(m_{\psi_{0}}, M_{Z_{D}}, \delta\right)$, the WMAP constraints reduce it to two $\left(M_{Z_{D}}, \delta\right)$.

We show in Fig.1 the points respecting WMAP, and the DAMA/LIBRA (with and without channeling) CoGeNT and $\mathrm{CRESST}^{1}$ results at $90 \%$ of CL. All the constraints have been calculated for a standard Maxwellian velocity distribution (with mean velocity $v_{0}=230 \mathrm{~km} / \mathrm{s}$ and an escape velocity $v_{\text {esc }}=600 \mathrm{~km} / \mathrm{s}$ ). One can observe in Fig. 1 that for all experiments, the regions are quite surprisingly near and correspond to $10 \mathrm{GeV} \lesssim M_{Z_{D}} \lesssim 30 \mathrm{GeV}$ and $10^{-4} \lesssim \delta \lesssim 10^{-3}$, which is in complete agreement with the measurement of electroweak precision tests. Moreover, such values of $\delta$ are typical of one loop-order corrections and can easily be generated by heavy-fermions loops in the $Z-Z_{D}$ propagator.

\section{Anomalies and gamma-ray line}

It is well known that any extension of the SM which introduces chiral fermions with respect to gauge fields suffers from anomalies, a phenomenon of breaking of gauge symmetries of the classical theory at one-loop level. Anomalies are responsible for instance for a violation of unitarity and make a theory inconsistent $[18,19]$. For this reason if any construction introduces a new fermionic sector to address the DM issue of the SM, it is vital to check the cancelation of anomalies and its

\footnotetext{
${ }^{1}$ For the CRESST estimation, we used an extrapolation given in the talk of T. Schwetz and the CRESST collaboration [17].
} 
$\mathrm{E}^{2} \mathrm{dN} / \mathrm{dE}$
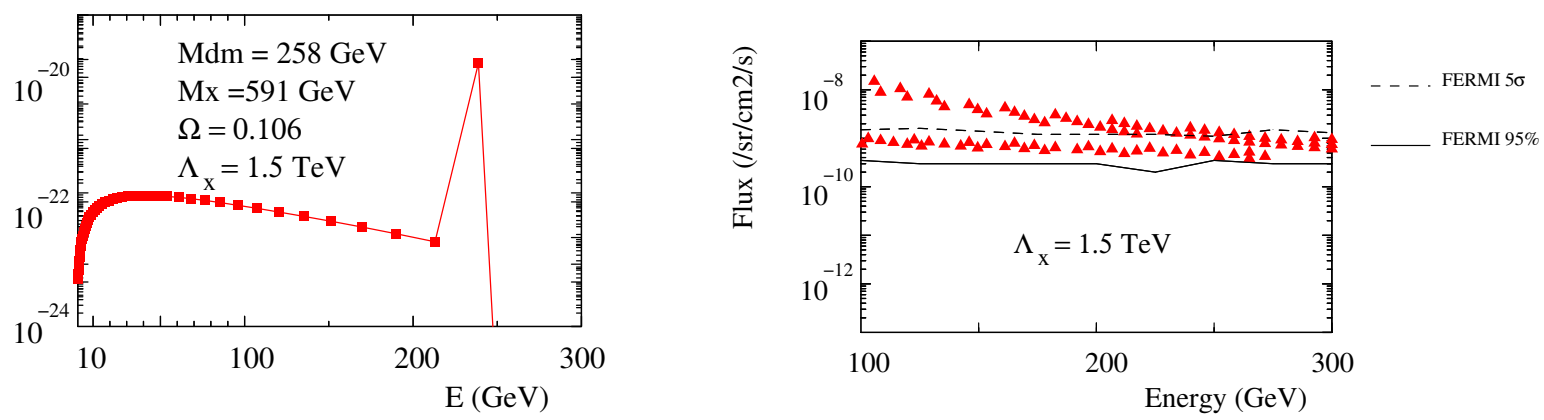

Figure 2: Left: example of gamma-ray flux respecting WMAP constraint for a DM mass of $258 \mathrm{GeV}$. Right: monochromatic $\gamma$-ray fluxes generated by anomaly-cancelation mechanism in comparison with expected $5 \sigma$ and $95 \%$ CL sensitivity contours ( 5 years of FERMI operation) for the conventional background and unknown WIMP energy, for an effective scale $\Lambda_{X}=1.5 \mathrm{TeV}$

consequences on the Lagrangian and couplings. The idea is to add to the Lagrangian local gauge non-invariant terms in the effective action whose gauge variations cancel the anomalous triangle diagrams. There exist two kinds of term which can cancel the mixed $U(1)_{D} \times G_{A}^{\mathrm{SM}}$ anomalies, with $G_{A}^{\mathrm{SM}}$ being one of the SM gauge group $S U(3) \times S U(2) \times U_{Y}(1)$ : the Chern Simons (CS) term which couples the $G_{A}^{\mathrm{SM}}$ to the $U(1)_{D}$ gauge boson, and the Peccei-Quinn (PQ, or Wess-Zumino (WZ)) term which couples the $G_{A}^{\mathrm{SM}}$ gauge boson to an axion. In the effective action, these terms are sometimes called Generalized Chern-Simons (GCS) terms [5]:

$$
\begin{aligned}
& \mathscr{L}_{i n v}=-\frac{1}{4 g^{\prime 2}} F^{Y \mu \nu} F_{\mu \nu}^{Y}-\frac{1}{4 g_{X}^{2}} F^{X \mu v} F_{\mu \nu}^{X}-\frac{1}{2}\left(\partial_{\mu} a_{X}-M_{X} X_{\mu}\right)^{2}-i \bar{\psi} \gamma^{\mu} D_{\mu} \psi \\
& \mathscr{L}_{v a r}=\frac{C}{24 \pi^{2}} a_{X} \varepsilon^{\mu \nu \rho \sigma} F_{\mu \nu}^{Y} F_{\rho \sigma}^{Y}+\frac{E}{24 \pi^{2}} \varepsilon^{\mu \nu \rho \sigma} X_{\mu} Y_{v} F_{\rho \sigma}^{Y} .
\end{aligned}
$$

The Stueckelberg axion $a_{X}$ ensures the gauge invariance of the effective Lagrangian and $g_{X}$ and $F_{\mu \nu}^{X}=\partial_{\mu} X_{v}-\partial_{v} X_{\mu}$ are the gauge coupling and field strength of $U(1)_{D}$. The axion has a shift transformation under $U(1)_{D}$

$$
\delta X_{\mu}=\partial_{\mu} \alpha \quad, \quad \delta a_{X}=\alpha M_{X} .
$$

The $\mathscr{L}_{\text {var }}$ will generate after the $S U(2) \times U(1)_{Y}$ tri-vectorial couplings $Z_{D} Z Z$ and $Z_{D} \gamma Z(Z \gamma \gamma$ coupling being forbidden by spin- momentum conservation). This generates new annihilation processes $\psi_{0} \psi_{0} \rightarrow Z_{D} \rightarrow Z Z / Z \gamma$ which can be observable through the only monochromatic gammaray line with energy $E_{\gamma}=m_{\psi_{0}}\left(1-\frac{M_{Z}^{2}}{4 m_{\psi_{0}}^{2}}\right)$ [12, 13]. Other models predicts several lines [20, 14], but none of them just one line.

As an illustrative point, we show in the left panel of Fig.2 an example of spectrum from the centre annulus that could be observable by the FERMI telescope, generated by DM annihilation within the pole region respecting WMAP constraint $\left(m_{\psi_{0}}=258 \mathrm{GeV}\right.$ and $\left.M_{Z_{D}}=591 \mathrm{GeV}\right)$. We can clearly distinguish a $\gamma$-ray line centered around $E_{\gamma}=m_{\psi_{0}}\left(1-\frac{M_{Z}^{2}}{4 m_{\psi_{0}}^{2}}\right)$ above the continuous 
flux produced by the annihilation process $\psi_{0} \psi_{0} \rightarrow Z Z / Z \gamma$. The expected sensitivity of FERMI telescope after 5 years of data taking is presented in the right panel of Fig.2.

We clearly see in the right panel of Fig.2 that for an effective scale $\Lambda_{X}=1.5 \mathrm{TeV}$ (scale of the "new physics" corresponding to the fermions generating the anomalies), all the parameter space would be observable by FERMI at $95 \% \mathrm{CL}$. Indeed, the points that respect the WMAP constraints lie around the pôle $M_{Z_{D}} \sim 2 m_{\psi_{0}}$ where $\sim 60 \%$ of the annihilation rate is dominated by the $Z \gamma$ final state. This proportion still holds for annihilating DM in the Galactic halo and gives a monochromatic line observable by FERMI.

\section{Conclusion}

We showed that the existence of a dark $U(1)_{D}$ gauge sector which interacts with the Standard Model only through its kinetic mixing or anomaly-generated couplings possesses a valid dark matter candidate respecting accelerator, cosmological and the more recent direct detection constraints. Moreover, considering the latest results of DAMA/LIBRA, CoGENT and CRESST, we demonstrated that a specific range of the kinetic mixing $\left(\delta \sim 10^{-4}-10^{-3}\right)$ can explain all these excesses for a dark boson mass $M_{Z_{D}} \sim 10-20 \mathrm{GeV}$, whereas anomaly cancelationconditions generate a monochromatic $\gamma$-ray line from DM annihilation into $Z \gamma$. Such a signature would be a smoking gun signal for these types of constructions It is interesting to notice that other constraints, coming from synchrotron radiation [21] or difuse gamma-ray emission [22] can give more restriction to the analysis.

\section{Acknowledgements}

Y.M. wants to thank particularly E. Dudas, T. Schwetz, G. Belanger, N. Fornengo and A. Romagnoni for useful discussions. The work was supported by the french ANR TAPDMS ANR-09JCJC-0146 and the spanish MICINNÕs Consolider-Ingenio 2010 Programme under grant MultiDark CSD2009-00064 and the E.C. Research Training Networks under contract MRTN-CT-2006035505. The author also wants to send all his regards to the "magic" organizing comitee.

\section{References}

[1] See e.g. P. Langacker, arXiv:0801.1345 [hep-ph]; M. Pospelov, A. Ritz and M. B. Voloshin, Phys. Lett. B 662 (2008) 53 [arXiv:0711.4866 [hep-ph]]; M. Pospelov, Phys. Rev. D 80 (2009) 095002 [arXiv:0811.1030 [hep-ph]]; M. Pospelov, A. Ritz and M. B. Voloshin, Phys. Lett. B 662 (2008) 53 [arXiv:0711.4866 [hep-ph]]; J. Kumar and J. D. Wells, Phys. Rev. D 74, 115017 (2006) [arXiv:hep-ph/0606183]; B. Batell, arXiv:1007.0045 [hep-ph].

[2] B. Holdom, Phys. Lett. B 166, 196 (1986); F. del Aguila, M. Masip and M. Perez-Victoria, Nucl. Phys. B 456 (1995) 531 [arXiv:hep-ph/9507455]; T. Cohen, D. J. Phalen, A. Pierce and K. M. Zurek, arXiv:1005.1655 [hep-ph].

[3] M. Baumgart, C. Cheung, J. T. Ruderman, L. T. Wang and I. Yavin, JHEP 0904 (2009) 014 [arXiv:0901.0283 [hep-ph]].

[4] E. J. Chun, J. C. Park and S. Scopel, arXiv:1011.3300 [hep-ph]. 
[5] P. Anastasopoulos, M. Bianchi, E. Dudas and E. Kiritsis, JHEP 0611 (2006) 057 [arXiv:hep-th/0605225].

[6] J. Kumar, A. Rajaraman and J. D. Wells, Phys. Rev. D 77 (2008) 066011 [arXiv:0707.3488 [hep-ph]]; I. Antoniadis, A. Boyarsky, S. Espahbodi, O. Ruchayskiy and J. D. Wells, arXiv:0901.0639 [hep-ph].

[7] R. Bernabei et al., Riv. Nuovo Cim. 26N1 (2003) 1 [arXiv:astro-ph/0307403]; R. Bernabei et al. [DAMA Collaboration], Eur. Phys. J. C 56 (2008) 333 [arXiv:0804.2741 [astro-ph]].

[8] C. E. Aalseth et al. [CoGeNT collaboration], arXiv:1002.4703 [astro-ph.CO].

[9] S. Andreas, C. Arina, T. Hambye, F. S. Ling and M. H. G. Tytgat, Phys. Rev. D 82 (2010) 043522 [arXiv:1003.2595 [hep-ph]]; S. Andreas, T. Hambye and M. H. G. Tytgat, JCAP 0810 (2008) 034 [arXiv:0808.0255 [hep-ph]].

[10] Y. Mambrini, JCAP 1009 (2010) 022 [arXiv:1006.3318 [hep-ph]].

[11] M. R. Buckley, D. Hooper and T. M. P. Tait, arXiv:1011.1499 [hep-ph].

[12] E. Dudas, Y. Mambrini, S. Pokorski and A. Romagnoni, JHEP 0908 (2009) 014 [arXiv:0904.1745 [hep-ph]].

[13] Y. Mambrini, JCAP 0912 (2009) 005 [arXiv:0907.2918 [hep-ph]].

[14] C. B. Jackson, G. Servant, G. Shaughnessy, T. M. P. Tait and M. Taoso, JCAP 1004 (2010) 004 [arXiv:0912.0004 [hep-ph]]; G. Bertone, C. B. Jackson, G. Shaughnessy, T. M. P. Tait and A. Vallinotto, arXiv:1009.5107 [astro-ph.HE].

[15] J. Goodman, M. Ibe, A. Rajaraman, W. Shepherd, T. M. P. Tait and H. B. P. Yu, Nucl. Phys. B 844 (2011) 55 [arXiv:1009.0008 [hep-ph]].

[16] D. N. Spergel et al. [WMAP Collaboration], Astrophys. J. Suppl. 170 (2007) 377 [arXiv:astro-ph/0603449]; E. Komatsu et al. [WMAP Collaboration], arXiv:0803.0547 [astro-ph].

[17] see talk by W. Seidel, WONDER 2010 Workshop, Laboratory Nazionali del Gran Sasso, Italy, March 22-23, 2010 and MPIK seminar by T. Schwetz, june21, 2010; T. Schwetz, arXiv:1011.5432 [hep-ph].

[18] D. J. Gross and R. Jackiw, Phys. Rev. D 6 (1972) 477; C. Bouchiat, J. Iliopoulos and P. Meyer, Phys. Lett. B 38 (1972) 519.

[19] C. Coriano, N. Irges and S. Morelli, JHEP 0707 (2007) 008 [arXiv:hep-ph/0701010].

[20] L. Bergstrom, P. Ullio and J. H. Buckley, Astropart. Phys. 9 (1998) 137 [arXiv:astro-ph/9712318]; T. Bringmann, L. Bergstrom and J. Edsjo, JHEP 0801, 049 (2008) [arXiv:0710.3169 [hep-ph]]. L. Bergstrom, T. Bringmann, M. Eriksson and M. Gustafsson, Phys. Rev. Lett. 94 (2005) 131301 [arXiv:astro-ph/0410359]; A. Birkedal, K. T. Matchev, M. Perelstein and A. Spray, arXiv:hep-ph/0507194; J. F. Beacom, N. F. Bell and G. Bertone, Phys. Rev. Lett. 94 (2005) 171301 [arXiv:astro-ph/0409403]; M. Gustafsson, E. Lundstrom, L. Bergstrom and J. Edsjo, Phys. Rev. Lett. 99 (2007) 041301 [arXiv:astro-ph/0703512].

[21] C. Boehm, J. Silk and T. Ensslin, arXiv:1008.5175 [astro-ph.GA]; D. Hooper and T. Linden, arXiv:1011.4520 [astro-ph.HE].

[22] C. Arina and M. H. G. Tytgat, arXiv:1007.2765 [astro-ph.CO]. 